\title{
PEREGRINE: AN ALL-PARTICLE MONTE CARLO CODE FOR RADIATION THERAPY
}

C. L. Hartmann Siantar, W. P. Chandler,

J. A. Rathkopf, M. M. Svatos, and R. M. White

Lawrence Livermore National Laboratory

University of California

P.O. Box 808

Livermore, CA 94550

This paper was prepared for submittal to

Proceedings of

International Conference on Mathematics

and Computations, Reactor Physics,

and Environmental Analyses

Portland, Oregon

April 30-May 4, 1995

September 1994

This is a preprint of a paperintended for publication in a joumal or proceedings. Since changes may be made before publication, this preprint is made available with the understanding that it will not be cited or reproduced without the permission of the author. 


\section{DISCLAIMER}

This document was prepared as an account of vork sponsored by an agency of the United States Government. Neither the United States Government nor the University of California nor any of their employees, makes any warranty, express or implied, or assumes any legal liability or responsibility for the accuracy, completeness, or usefulness of any information, apparatus, product, or process disclosed, or represents that its use would not infringe privately owned rights. Reference herein to any specific commercial products, process, or service by trade name, trademark, manufacturer, or otherwise, does not necessarily constitute or imply its endorsement, recommendation, or favoring by the United States Government or the University of California. The views and opinions of authors expressed herein do not necessarily state or reflect those of the United States Government or the University of California, and shall not be used for advertising or product endorsement purposes. 


\section{DISCLAIMER}

Portions of this document may be illegible in electronic image products. Images are produced from the best available original document. 


\title{
PEREGRINE: AN ALL-PARTICLE MONTE CARLO CODE FOR RADIATION THERAPY
}

\author{
C. L. Hartmann Siantar, W. P. Chandler, \\ J. A. Rathkopf, M. M. Svatos, and R. M. White \\ Lawrence Livermore National Laboratory \\ University of California \\ P.O. Box 808, Livermore, California 94550
}

\begin{abstract}
The goal of radiation therapy is to deliver a lethal dose to the tumor while minimizing the dose to normal tissues. To carry out this task, it is critical to calculate correctly the distribution of dose delivered. Monte Carlo transport methods have the potential to provide more accurate prediction of dose distributions than currently-used methods. PEREGRINE is a new Monte Carlo transport code developed at Lawrence Livermore National Laboratory for the specific purpose of modeling the effects of radiation therapy. PEREGRINE transports neutrons, photons, electrons, positrons, and heavy charged-particles, including protons, deuterons, tritons, helium-3, and alpha particles. This paper describes the PEREGRINE transport code and some preliminary results for clinically relevant materials and radiation sources.
\end{abstract}

\section{INTRODUCTION}

Every year in the United States, about 900,000 people develop a serious form of cancer. ${ }^{1}$ About a third of these patients die each year with active disease at and around the tumor site. Since radiation therapy targets specific areas of the body, improvement in radiation treatment techniques has the potential to significantly reduce both mortality and morbidity in a large number of patients. Radiation therapy is currently used to treat about $60 \%$ of all cancer patients. ${ }^{1}$

Radiation therapy is performed with several types of ionizing radiation. Approximately $80 \%$ of patients are treated with photons, ranging in maximum energy from $250 \mathrm{keV}$ to $25 \mathrm{MeV}$. The balance are treated primarily with electrons with energies from 4 to $25 \mathrm{MeV}$. In addition, there are more than 15 fast neutron therapy centers and 25 proton therapy facilities which have treated thousands of patients worldwide. Fast neutron therapy is performed with neutron energies up to $70 \mathrm{MeV}$, while proton therapy is performed with proton energies ranging from about 50 to $250 \mathrm{MeV}$.

The goal of radiation therapy is to deliver a lethal dose to the tumor while maintaining an acceptable dose level in surrounding sensitive structures. Because tumors and sensitive structures are often located in close proximity, accuracy in the calculation of dose distributions is critically important. Most current methods used to calculate dose in the body rely on interpolation schemes based on dose measurements made in a water phantom. Heterogeneities such as bone and airways are treated in a very approximate way or ignored altogether.

\section{THE PEREGRINE CODE}

The PEREGRINE code is designed to calculate dose in the body using three-dimensional Monte Carlo transport. Neutrons, protons, deuterons, tritons, helium-3, alpha particles, photons, electrons, and positrons are transported in a completely coupled manner, using the Monte Carlo All-Particle Method. ${ }^{2}$ This paper discusses the input options, physical data, and transport methods used in PEREGRINE, as well as preliminary results for monoenergetic photon and neutron beams.

Input to PEREGRINE consists of defining the transport mesh and the radiation source. The user can define the cartesian transport mesh either manually or from a computed tomography (CT) scan. Each zone within the mesh is assigned a material number which is associated with a specific atomic composition and density. If the transport mesh is obtained from a CT scan, the $x$ and $y$ zone dimensions are defined as the dimensions of each pixel on the CT slice, while the $z$ dimension is the spacing between CT slices. Materials are assigned using a thresholding algorithm based on ranges of $\mathrm{CT}$ numbers. 


\section{Front View}

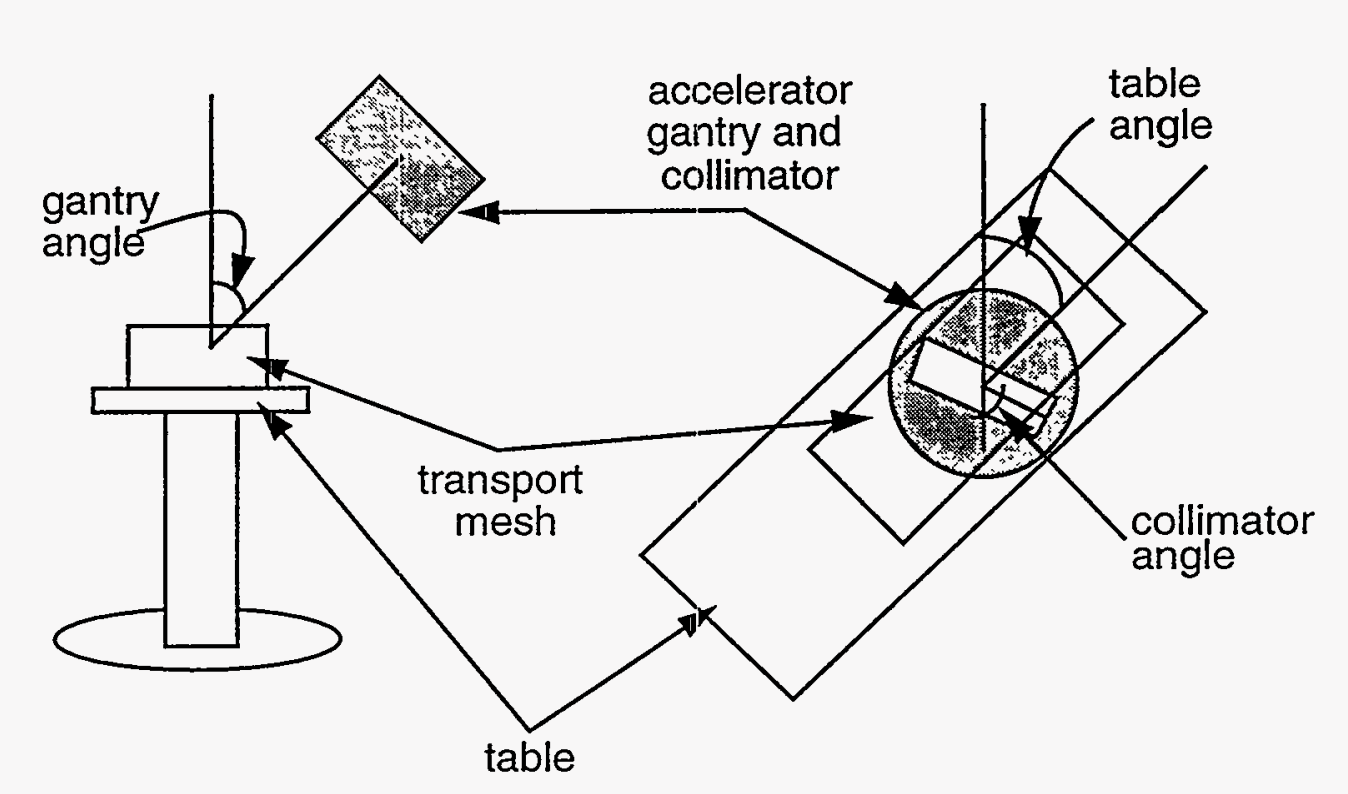

Top View

Figure 1. Schematic diagram showing the three angles (gantry, table, and collimator) for positioning the incident radiation beams.

The current material library includes individual elements, tissues tabulated by ICRU Report $46^{3}$, and materials commonly used in clinical dosimetry measurements. For photon, electron, and positron transport at all energies and neutron and heavy charged-particle transport below $20 \mathrm{MeV}$, materials may consist of ${ }^{1} \mathrm{H},{ }^{10} \mathrm{~B},{ }^{12} \mathrm{C},{ }^{14} \mathrm{~N},{ }^{16} \mathrm{O},{ }^{19} \mathrm{~F},{ }^{23} \mathrm{Na}$, ${ }^{{ }^{n a t}} \mathrm{Mg},{ }^{31} \mathrm{P},{ }^{32} \mathrm{~S},{ }^{\text {nat }} \mathrm{Cl},{ }^{\text {nat }} \mathrm{K},{ }^{\text {nat }} \mathrm{Ca}$, ${ }^{\text {nat }} \mathrm{Fe}$, and ${ }^{127} \mathrm{I}$. For neutron and heavy charged-particle transport above $20 . \mathrm{MeV}$, allowed elements are limited to ${ }^{1} \mathrm{H},{ }^{12} \mathrm{C},{ }^{14} \mathrm{~N},{ }^{16} \mathrm{O},{ }^{19} \mathrm{~F},{ }^{31} \mathrm{P}$, and ${ }^{40} \mathrm{Ca}$.

The radiation source can be defined as a complex beam or set of beams in a standard clinical isocentric gantry and table geometry. The user specifies the accelerator gantry, collimator, and table angles, as well as the $(x, y, z)$ location of the table/accelerator center of rotation (isocenter) in the transport mesh (see Figure 1). Source characteristics are described at a plane in space (usually outside the accelerator gantry assembly) that is defined to be perpendicular to the ray connecting the radiation source and the gantry/table isocenter, as shown in Figure 2. The energy and angular distributions of the radiation sources are chosen from libraries that include monoenergetic, monodirectional options as well as energy and angular distributions derived from Monte Carlo simulations of actual beam delivery systems. The field shape is defined as either a series of rectangles or concentric rings. Each rectangle or ring may have its own energy and angular distribution. The angular distribution chosen by the user modifies the trajectory of the particle at the beam definition plane. This scheme allows complex spatial and energy distributions in each incident beam.

\section{PHYSICAL DATA}

PEREGRINE uses the Monte Carlo All Particle Method (MCAPM) for particle transport. The MCAPM is a system of data and algorithms that enables the coupled Monte Carlo simulation of many particle types. Extensive evaluated data libraries have been developed to describe the interaction of neutrons, photons, electrons, and heavy charged-particles with matter. Each interaction may result in the production of any of these partieles. Additional data describing electron Coulomb interactions as well as the relaxation of ions as a function of vacancy position and the resulting emission of $\mathrm{x}$-rays or electrons through fluorescence and Auger processes are planned to be part of MCAPM, but are not yet implemented in PEREGRINE.

The continuous-energy LLNL data libraries are organized by incident particle type, and contain information describ-

$\therefore \quad \cdots$




\section{Front View}

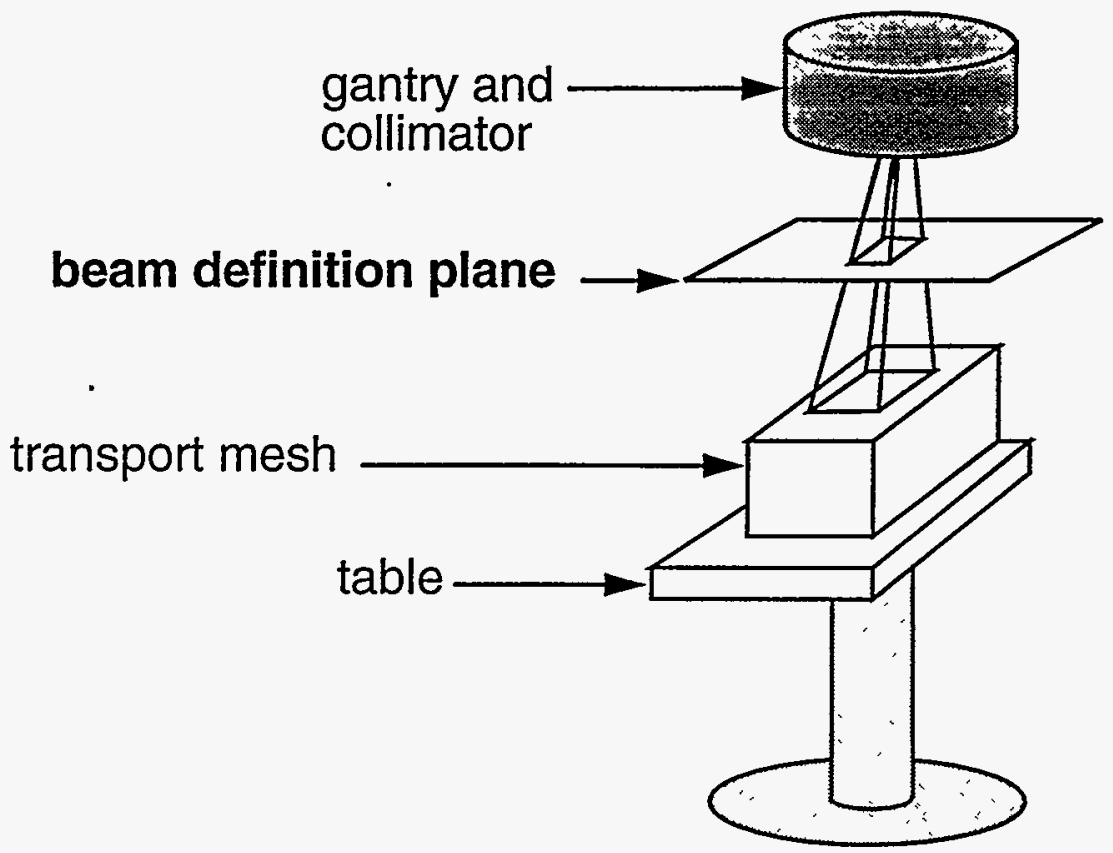

Figure 2. Schematic diagram illustrating the beam definition plane.

ing the attributes of all output particles. The processed neutron and heavy charged-particle cross sections are multigroup, and photon cross sections are pointwise tabulated using an energy grid that is the same for all target elements. There are 175 neutron energy groups, ranging in energy between $1.3 \times 10^{-3} \mathrm{eV}$ to $20 \mathrm{MeV}$, and 176 photon energy points at energies from $100 \mathrm{eV}$ to $30 \mathrm{MeV}$. Heavy charged-particle nuclear scattering and large angle Coulomb cross sections are tabulated in 63 energy groups spanning $1.0 \times 10^{-4} \mathrm{eV}$ to $20 \mathrm{MeV}$. Continuous electron, positron, and heavy charged-particle slowing-down data extend to $1000 \mathrm{MeV}$. We are combining the current cross section and continuous slowing-down data with neutron and proton nuclear cross sections from the new LLNL high energy data libraries that extends to $250 \mathrm{MeV}^{3,4}$ This will allow PEREGRINE to carry out accurate Monte Carlo transport calculations for fast neutron and proton therapy.

\section{MONTE CARLO TRANSPORT METHODS}

PEREGRINE carries out Monte Carlo transport calculations as follows. First, a particle is selected from the radiation source. Depending on its type, it is passed to the appropriate neutron, photon, electron, positron, or heavy charged-particle transport package, where it is tracked until it undergoes a collision. Each nuclear reaction and photon interaction is handled by calling the MCAPM reaction physics package. Given an incident particle, this package returns the energies and angles of all secondary particles resulting from the collision. These particles are stored in the secondary bank. Control is then returned to the Monte Carlo tracker, which selects a particle from the secondary bank or, when the bank is exhausted, from the radiation source. This process continues until all particles in the source and secondary bank have been followed.

Although neutron and heavy charged-particle cross sections are multigroup, each particle possesses a discrete energy value, allowing the collisionkinematics.tobe performed en a continuous energy basis. This Plechaty Hybrid ${ }^{3}$ scheme exploits the strengths of both the pure continuous energy method and the pure multi-group method-a smaller cross section database and accurate kinematics.

Neutrons and photons are transported using standard analog Monte Carlo methods. Energy transferred to particles that are not being tracked is deposited locally, with expected energy deposition providing variance reduction. The minimum neutron energy for tracking is $1.0 \times 10^{-8} \mathrm{MeV}$, while the minimum photon energy is $1.0 \times 10^{-4} \mathrm{MeV}$. 
For heavy charged-particles, nuclear reactions and large-angle Coulomb scattering are treated on an event-by-event basis, while all other Coulomb interactions are accounted for with the condensed history method. ${ }^{6}$ Charged particle stopping powers are taken from the tabulations of Anderson and Ziegler. ${ }^{7}$ In order to account for the multiple Coulomb collisions that occur within a condensed history step, the particle's energy and trajectory are modified using Gaussian distributions with variances determined from the Rutherford scattering cross section (energy straggling) and from the Highland approximation to a Moliere distribution (multiple scattering). ${ }^{8}$ The minimum energy for heavy charged-particle energy tracking is $0.1 \mathrm{MeV}$.

Electron and positron transport is currently accomplished using the condensed history method. Electron and positron stopping powers are taken from ICRU Report $37 .^{9}$ The electron's (or positron's) energy and trajectory are modified using Gaussian distributions with variances determined from the Møller scattering cross section (energy straggling) and from a Gaussian fit to a Moliere distribution ${ }^{10}$ (multiple scattering). Bremsstrahlung photons are produced along each condensed history step. A Russian rouletting scheme applied to photon production minimizes the number of bremsstrahlung photons. The minimum electron and positron energy tracked is $0.01 \mathrm{MeV}$. In the future, PEREGRINE will use the Macro Response Monte Carlo Method ${ }^{11}$ as an alternative to condensed history.

\section{RESULTS}

To benchmark PEREGRINE for use in the clinic, we have begun by comparing it with other, well-tested Monte Carlo codes. This section shows the results of preliminary code comparisons for photon and neutron beams incident on simple phantoms constructed of biologically relevant materials.

In Figure 3, we compare PEREGRINE results with those of EGS4/PRESTA ${ }^{12}$ for photon pencil beams incident on water. For PEREGRINE calculations, photons were incident on the center of the $100 \times 100 \mathrm{~cm}^{2}$ face of a $12 \mathrm{~cm}$-long slab rectangular phantom. For the EGS4 calculations, photons were incident on the $113 \mathrm{~cm}$-diameter face of a $12 \mathrm{~cm}$ long slab cylindrical phantom. Because the phantoms for the PEREGRINE and EGS4 calculations are so wide, the difference between calculations done in cartesian and cylindrical geometry is insignificant. Photons, electrons, and positrons were tracked. Statistical errors are less than $\sim 1 \%$. PEREGRINE and EGS4 agree well in the buildup areas near the entrance of the phantom. This suggests that current PEREGRINE electron/positron transport methods are sufficiently accurate for photon beams incident on water, even though the PEREGRINE condensed history transport algorithms are less sophisticated than those used in EGS4. At depths greater than $4 \mathrm{~cm}$, PEREGRINE and EGS4 agree well for 5 and $10 \mathrm{MeV}$ photon energies, but differ by $5-6 \%$ for the 15 and $20 \mathrm{MeV}$ beams. We are currently investigating these discrepancies.

We have also carried out calculations with a slab phantom consisting of fat, bone, and muscle. Table 1 summarizes the composition and density assumed for each material. For these calculations, neutron pencil beams are incident on the center of the $10 \times 10 \mathrm{~cm}^{2}$ face of the $20 \mathrm{~cm}$-long phantom. Figure 4 shows the results of calculations in which we compared PEREGRINE with TART ${ }^{13}$ and $\mathrm{MCNP}^{14}$ for neutron pencil beams incident on a mesh consisting of generic fat, bone and muscle (see Table 1). All three codes tracked neutrons and photons, depositing the energy given to all other particles locally. Absorbed dose was tabulated for each zone. MCNP calculations were done using both the ENDL ${ }^{15}$ and ENDF/B-V ${ }^{16}$ nuclear data libraries. PEREGRINE and TART used the ENDL nuclear data library. Statistical errors are less than 1\%. Results show that PEREGRINE, TART, and MCNP/ENDL are in good agreement for the energies studied. However, for 15 and $20 \mathrm{MeV}$ neutron energies, MCNP calculations made with the ENDF library result in significantly higher absorbed doses than those relying on the ENDL database. Differences in the databases are currently being investigated.

Further calculations, plotted in Figure 5, show PEREGRINE results for the same neutron energies, but this time tracking secondary protons and alpha particles in addition to neutrons and photons. These calculations indicate significant buildup regions at the air-fat and bone-muscle interfaces. Particle-dependent dose calculations show that these effects are related to the distribution of dose deposited by protons created in the phantom. Reaction tallies indicate that this is caused by neutron scattering on hydrogen, which occurs more often in fat and muscle than in bone, proportional to the number of hydrogen atoms per zone in each material. Scattered protons deposit their energy forward of the collision site. The lack of protons emerging from the bone causes the depression at the bone-muscle interface. 


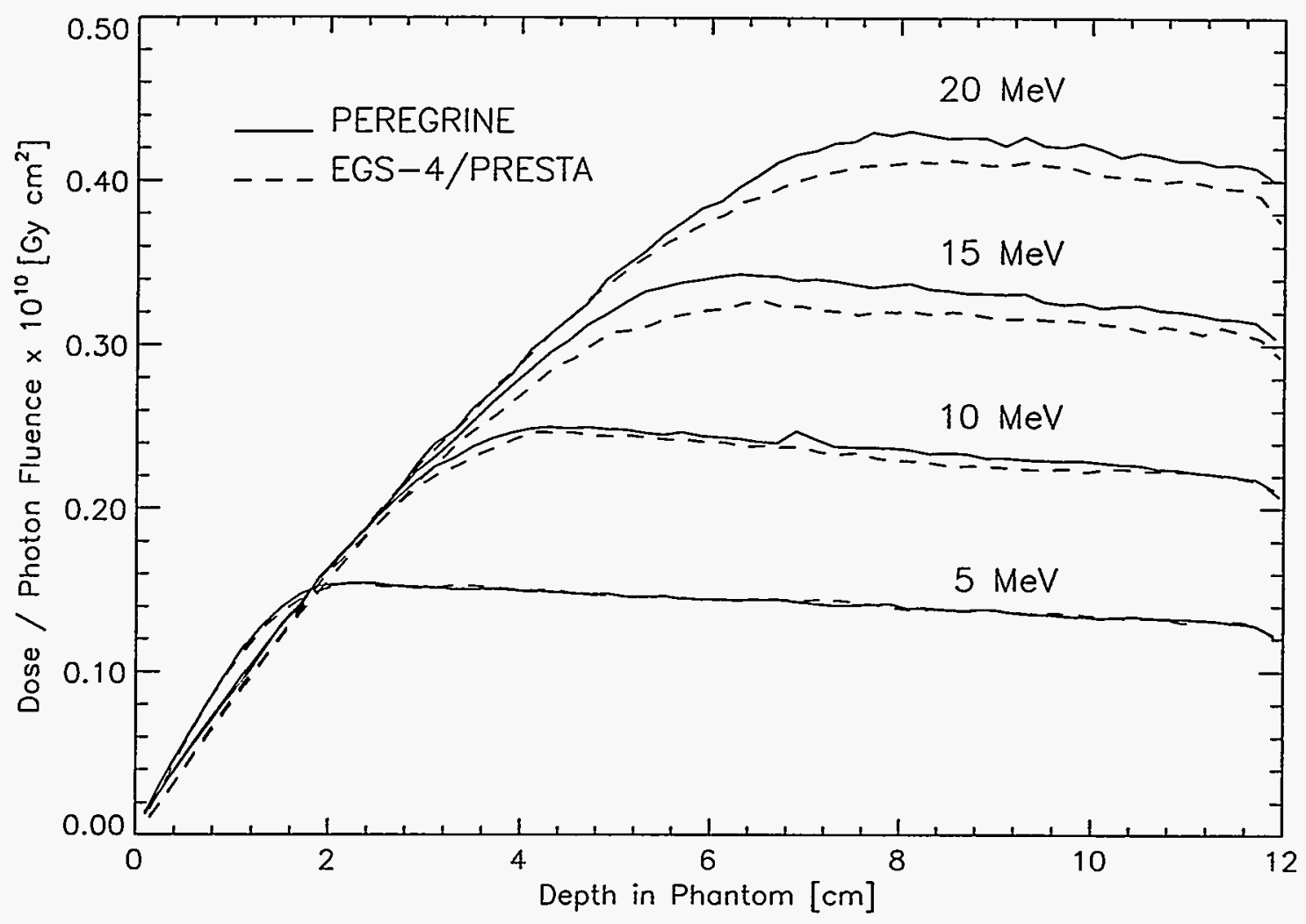

Figure 3. PEREGRINE and EGS4/PRESTA transport calculations for $5,10,15$, and $20 \mathrm{MeV}$ photon pencil beams incident on a water phantom. Photons, electrons, and positrons were tracked.

\begin{tabular}{|c|c|l|l|}
\hline & Fat & Bone & Muscle \\
\hline \hline${ }^{1} \mathrm{H}$ & $12.0 \%$ & $4.7 \%$ & $10.3 \%$ \\
\hline${ }^{12} \mathrm{C}$ & $63.9 \%$ & $14.5 \%$ & $11.7 \%$ \\
\hline${ }^{14} \mathrm{~N}$ & $0.8 \%$ & $4.2 \%$ & $3.2 \%$ \\
\hline${ }^{16} \mathrm{O}$ & $23.3 \%$ & $44.9 \%$ & $74.8 \%$ \\
\hline${ }^{31} \mathrm{P}$ & - & $10.6 \%$ & - \\
\hline${ }^{\text {nat }} \mathrm{Ca}$ & - & $21.1 \%$ & - \\
\hline \hline Density & $0.92 \mathrm{~g} / \mathrm{cm}^{3}$ & $1.85 \mathrm{~g} / \mathrm{cm}^{3}$ & $1.07 \mathrm{~g} / \mathrm{cm}^{3}$ \\
\hline
\end{tabular}

Table 1. Mass fractions and densities for elements used in neutron beam calculations. 


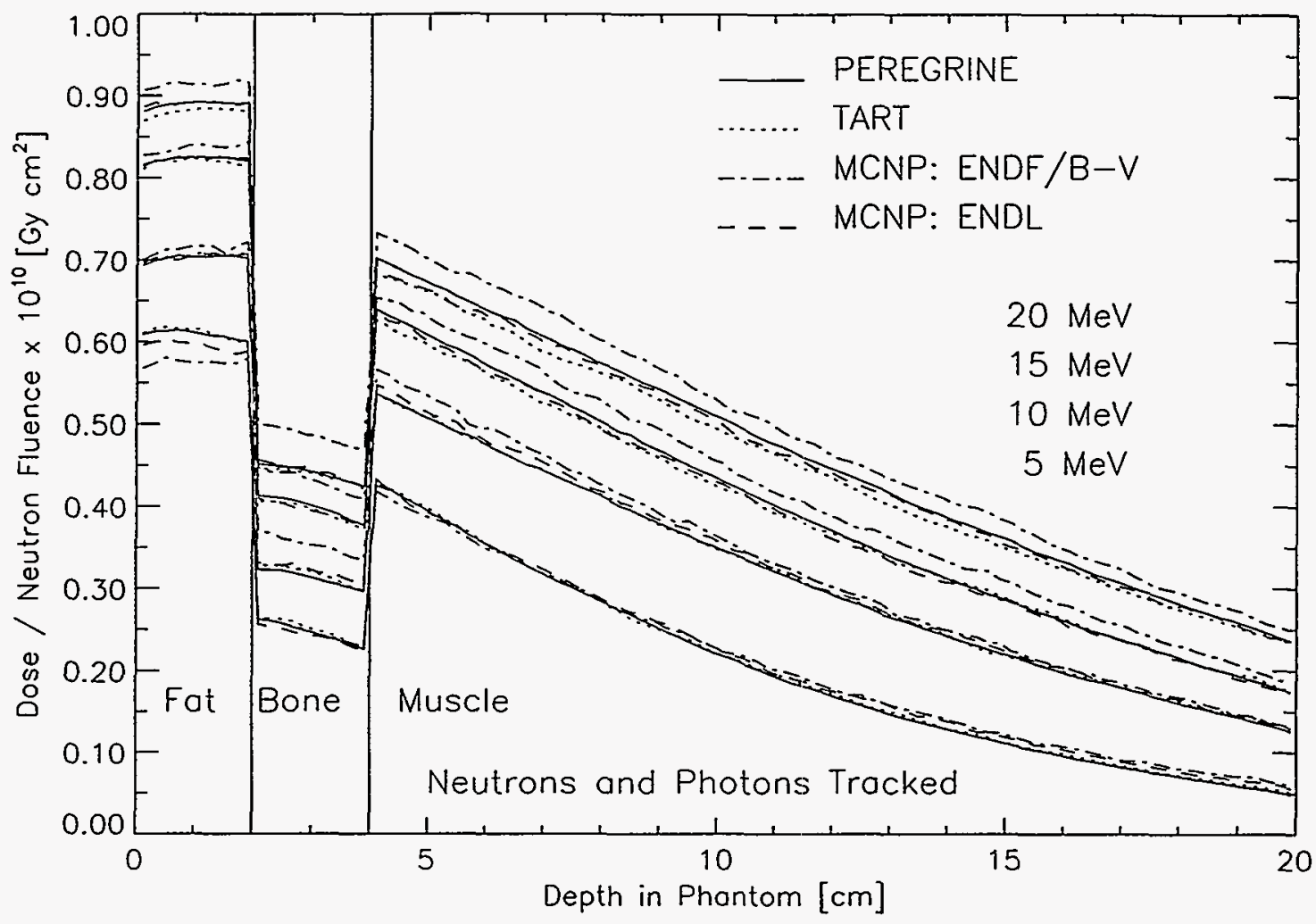

Figure 4. PEREGRINE, TART, and MCNP transport calculations for 5, 10, 15, and $20 \mathrm{MeV}$ neutron pencil beams incident on a slab phantom consisting of fat, bone, and muscle. Neutrons and photons were tracked, while energy from all other reaction products was deposited locally.

Figure 6 shows the results of calculations done for a $14.1 \mathrm{MeV}$ neutron beam incident on the left anterior surface of a human chest. The transport mesh, determined from a CT scan of a chest, was irradiated with a $5 \times 5 \mathrm{~cm}^{2}$ rectangular, 14.1-MeV monoenergetic, parallel neutron beam. 90, 80, 70, 60, 50, 25 and 1 percent isodose lines are indicated.The materials assigned to the $\mathrm{CT}$ mesh in this problem were muscle and bone, with compositions described in Table 1 , and dry air and lung (adult - healthy, inflated), with compositions taken from Reference 2. The number of transport zones in the $x, y$, and $z$ direction are 320,320, and 29, respectively. The $x, y$, and $z$ zone dimensions are 1.3,1.3, and $5.0 \mathrm{~mm}$. This problem represents a three-dimensional case with almost three million zones. Ten million incident neutrons comprised the radiation source. Neutrons and photons were tracked, with the energy transferred to charged particles deposited locally. Currently, PEREGRINE can calculate 14.0 incident neutrons/s in a coupled mode on a Sun Microsystems Sparc-10 computer with $128 \mathrm{Mb}$ of memory. Effort to date has focused on Monte Carlo transport code design and implementation, nuclear data modeling, and benchmarking, with minimal consideration to code optimization.

\section{CONCLUSIONS}

PEREGRINE is a new, all-particle Monte Carlo transport code developed at Lawrence Livermore National Laboratory for the specific purpose of modeling the effects of radiation therapy. It is written almost entirely in FORTRAN77 with a small portion in C, and is designed to run on medern LDNEX workstations: Preliminary sesults show that PEREGRINE is in good agreement with other, well-benchmarked codes for neutron and photon beams incident on simple slab phantoms constructed of biologically relevant materials. Future efforts will focus on comparing PEREGRINE with other codes for more complex geometries and benchmarking PEREGRINE against clinical radiation beam measurements. 


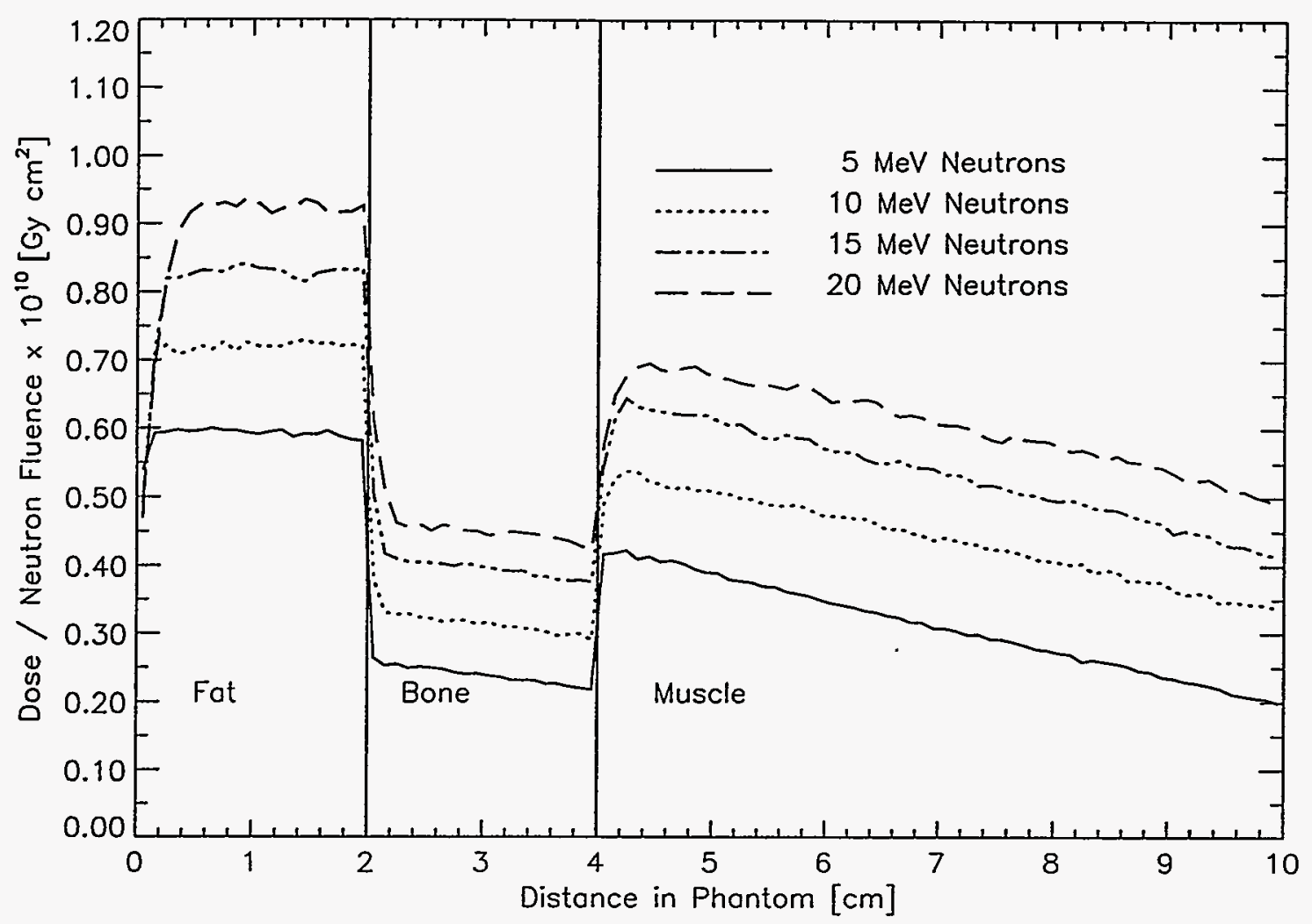

Figure 5. PEREGRINE results for $5,10,15$, and $20 \mathrm{MeV}$ neutron pencil beams incident on a slab phantom consisting of fat, bone, and muscle. Neutrons, photons, protons, and alpha particles were tracked, while energy from all other reaction products was deposited locally. 


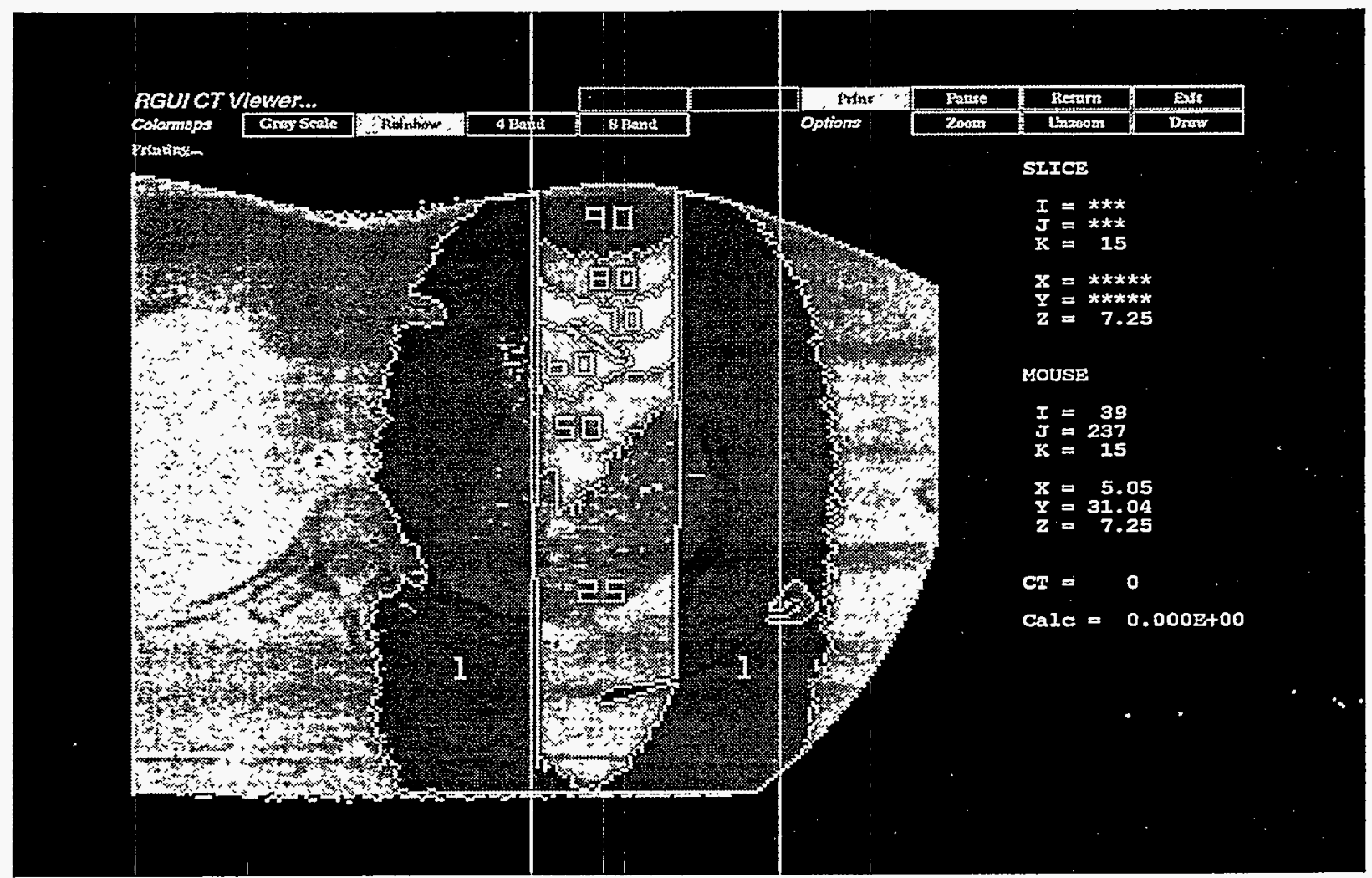

Figure 6. CT slice of a chest with a $5 \times 5 \mathrm{~cm}^{2}$ rectangular, $14.1 \mathrm{MeV}$ monoenergetic parallel neutron beam incident on the left lung. The 90, 80,70,60,50,25, and 1 percent isodose curves are shown as calculated by the three-dimensional, all-particle Monte Carlo PEREGRINE code.

\section{ACKNOWLEDGEMENTS}

The authors would like to thank M. B. Chadwick, D. E. Cullen, L. J. Cox, E. Plechaty, and D. A. Resler for many helpful discussions. This work was performed under the auspices of the U.S. Department of Energy by the Lawrence Livermore National Laboratory under contract no. W-7405-ENG-48.

\section{REFERENCES}

1. C. A. Perez and L. W. Brady, Principals and Practice of Radiation Oncology, J. P. Lippincott, Philadelphia, 1992.

2. J.A. Rathkopf, "MCAPM: All Particle Method Generator and Collision Package," UCRL-112310, Lawrence Livermore National Laboratory, Livermore, CA (1992).

3. ICRU Report 46, Photon, Electron, Proton, and Neutron Interaction Data for Body Tissues, International Commission on Radiation Units and Measurements, Bethesda, MD (1992).

4. M. B. Chadwick, M. Blann, P. G. Young, and G. Reffo, "Model Calculations of Nuclear Data for Biologically-Important Elements," Proceedings of the International Conference on Nuclear Data for Science and Technology, J. K. Dickens, ed., Gatlinburg, TN, May 9-13, 1994, in press. 
5. L. J. Cox, M. B. Chadwick and D. A. Resler, "New Nuclear Data for High-energy All-Particle Monte Carlo Transport," Proceedings of the International Conference on Nuclear Data for Science and Technology, J. K. Dickens, ed., Gatlinburg, TN, May 9-13, 1994, in press.

6. M. J. Berger, "Monte Carlo Techniques in Medical Radiation Physics," Phys. Med. Biol. 36861 (1991).

7. H. H. Anderson and J. F. Ziegler, Hydrogen, Stopping Powers and Ranges in All Elements, Vol. 3 of The Stopping and Ranges of Ions in Matter, organized by J. F. Ziegler, Pergamon Press, New York, NY (1977).

8. V. L. Highland, "Some Practical Remarks on Multiple Scattering," Nucl. Inst. Meth. 161,171 (1979).

9. ICRU Report 37, Stopping Powers for Electrons and Positrons, International Commission on Radiation Units and Measurements, Bethesda, MD (1984).

10. W.T. Scott, "The Theory of Small-Angle Multiple Scattering of Fast Charged Particles," Rev. Mod. Phys. 35 2, 231 (1963).

11. M. M. Svatos, T. R. Mackie, C. T. Ballinger, W. P. Chandler, C. L. Hartmann Siantar, and J. A. Rathkopf, "Electron Transport in Radiotherapy Using Look Up Monte Carlo," this conference.

12. A. F. Bielajew, H. Hirayama, W. R. Nelson, and D. W. O. Rogers, "History, Overview and Recent Improvements of EGS4," Report NRC-PIRS-0436 Ottawa, Canada (1994).

13. A. Edwards, J. Kimlinger and E. Plechaty, "TART Input Manual," UCRL-17026 Rev. 3.0.3, Lawrence Livermore National Laboratory, Livermore, CA (1992).

14. J. F. Briesmeister, "MCNP - A General Monte Carlo n-Particle Transport Code - Version 4A," LA12625 Los Alamos National Laboratory, Los Alamos, NM (1988).

15. "The LLL Evaluated-Nuclear-Data Library (ENDL) for Z=0-98" Volume 15, Part A in the UCRL50400 series, An Integrated System for Production of Neutronics and Photonics Calculational Constants, data files revised 1994, Lawrence Livermore National Laboratory, Livermore, CA (1978).

16. "ENDF-102 Data Formats and Procedures for the Evaluated Nuclear Data File, ENDF/B-VI," BNL-NCS-50496, revised, November, 1983, National Nuclear Data Center, Brookhaven National Laboratory, Upton, NY (1991). 